\title{
IV. Anleitung des Nationalkomitees „Freies Deutschland“ durch das Institut 99
}

\begin{abstract}
Das Nationalkomitee „Freies Deutschland“ war bis Kriegsende die tragende Säule der Frontpropaganda der Roten Armee. Ebenso war es in den Kriegsgefangenenlagern bis zu seiner Auflösung Ende 1945 als „Bewegung Freies Deutschland“ das gültige Erziehungskonzept. Dem Institut 99 fiel die Aufgabe zu, das propagandistische Auftreten des Nationalkomitees mit den politischen Zielen der Sowjetunion abzustimmen. Sein unmittelbarer Einfluß beschränkte sich allerdings auf die Redaktionsstäbe des NKFD in Moskau. Das Institut 99 versuchte daher, durch das Entsenden von „Bevollmächtigten des NKFD“ direkten Einfluß auf die Propaganda in den Kriegsgefangenenlagern und an den Fronten zu nehmen. Dabei mußte es mit UPVI (in den Lagern) und GlavPURKKA (an den Fronten) kooperieren.
\end{abstract}

\section{Redaktionen im Institut 99}

Zeitungen für Kriegsgefangene gab es in den sowjetischen Lagern seit dem Herbst 1941. Diese wurden in der Regel von kommunistischen Emigranten gemacht, die einer entsprechenden Unterabteilung der 7. Verwaltung des GlavPURKKA unterstellt waren. Im September 1943 wurden diese Redaktionsstäbe vom Institut 99 übernommen. Dort arbeiteten nun nicht nur die Redaktionen des Nationalkomitees „Freies Deutschland“, sondern auch die anderer Kriegsgefangenenzeitungen. Die deutsche war allerdings mit Abstand die größte unter ihnen. Die italienische Zeitung „Alba" hatte vier, die ungarische Zeitung „Wort der Wahrheit“ drei, später fünf, und die rumänische „Freie Stimme“ hatte ebenfalls erst drei, dann fünf Mitarbeiter. Demgegenüber verfügte die Redaktion des „Freien Deutschland“ von Anfang an über zehn Mitarbeiter. Zudem gab es die Radioredaktion des Senders „Freies Deutschland“, die sich von anfangs neun auf fünfzehn Mitarbeiter vergrößerte ${ }^{1}$. Schon allein durch die personelle Ausstattung wird deutlich, daß sich die Arbeit der Redaktionen im Institut 99 vornehmlich auf die deutschen Kriegsgefangenen bzw. die Radiohörer im Deutschen Reich konzentrierte. Wolfgang Leonhard, der in den Redaktionen des NKFD arbeitete, beschrieb die Ausstattung der anderen Redaktionen im Institut 99 mit den Worten: „Die Zimmer waren kleiner und wirkten gegenüber denen des Nationalkomitees wie die armen Verwandten.“2

Die Zeitung „Freies Deutschland“ löste das seit Ende Oktober 1941 erscheinende Kriegsgefangenenblatt „Das Freie Wort“ ab. Im Stil hatte das „Freie Wort“ mehr ei-

1 Geschäftsverteilungspläne des Instituts 99 vom 1. 10. 1943 und vom 1. 9. 1944, RGASPI 578/5/4, Bl. 5-6 und 495/77/39, Bl. 1-2; siehe Anlage Nr. 1.

2 Leonhard, Revolution, S. $284 \mathrm{f}$. 
ner Frontzeitung der Roten Armee entsprochen. Inhaltlich hatten sich die Artikel auf die „Entlarvung des volksfeindlichen Charakters des in Deutschland herrschenden faschistischen Regimes und der nazistischen Ideologie" und die "Verbreitung der Wahrheit über den Sozialismus und die Sowjetunion“ konzentriert ${ }^{3}$. Das „Freie Deutschland" dagegen verstand sich - so der Untertitel im Kopf der Zeitung - als „Organ des Nationalkomitees Freies Deutschland“. Es richtete sich in erster Linie an die Aktivisten der Bewegung in den Lagern. Aus dieser Zielsetzung leitete Weinert $\mathrm{ab}$, „daß sie nicht in volkstümlichem Ton gehalten wurde, sondern politisch reifere Soldaten und gebildete Offiziere als Leser im Auge hatte" ${ }^{4}$. Die Aufmachung und der journalistische Stil des „Freien Deutschland“ entsprachen dem einer politischen Tageszeitung 5 . Neben der Zeitung „Freies Deutschland“, die sich in erster Linie an die Anhänger des NKFD richtete, gab es den Sender „Freies Deutschland“. Er fungierte als „Sprachorgan des Nationalkomitees für die breiteste Öffentlich-

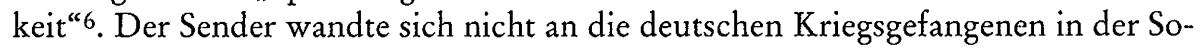
wjetunion - die ihn bestenfalls über den Lagerfunk hätten hören können -, sondern an die deutschen Frontsoldaten und die deutsche Zivilbevölkerung ${ }^{7}$. Er wurde auf Mittel- und Kurzwelle, nach dem Kriegsende auch auf Langwelle ausgestrahlt mit anfangs vier, ab Mitte 1944 täglich acht Sendungen. Allerdings war die Empfangsqualität in Deutschland wegen starker Störsender sehr schlecht ${ }^{8}$. In Moskau dagegen war der Sender „Freies Deutschland“ nicht zu hören”.

Die Redaktionsstäbe des NKFD im Institut 99 waren mit deutschen Kommunisten besetzt, von denen einige bereits für die 7. Verwaltung des GlavPURKKA gearbeitet hatten. Die Zeitungsredaktion blieb bis zum Kriegsende in ihrer personellen Zusammensetzung konstant. Sie bestand aus vier Redakteuren: Rudolf Herrnstadt (Chefredakteur), Alfred Kurella (Nachrichtenressort), Karl Maron (Militärressort) und Lothar Bolz (Deutschlandressort). Als Volontäre arbeiteten Ernst Held und Wolfgang Leonhard („Vladimir") in der Redaktion mit. Leonhard wechselte im Mai 1944 zum Sender „Freies Deutschland“ und wurde durch Peter Florin ersetzt. Die Zeitungsredaktion verfügte außerdem über drei deutsche (Gertrud Steier, Erna Seiler und Dora Gordeeva) und zwei russische Schreibkräfte bzw. technische Mitarbeiterinnen ${ }^{10}$. Die Radioredaktion unterlag, bedingt durch ihre Erweiterung, stärkeren Veränderungen. Chefredakteur war bis Mai 1945 Anton Ackermann, der anfangs drei Redakteure unter sich hatte: Hans Mahle, Willi Bredel und Kurt Fischer. Zum Jahreswechsel 1943/44 kamen Max Keilson und Fritz Erpenbeck hinzu. Nach dem Ausscheiden von Willi Bredel, der im Frühjahr 1944 zur sogenannten Broschürenredaktion im Institut 99 wechselte, wurde der Redaktionsstab des Senders durch Georg Schneider, Gustav von Wangenheim und Eleonore

3 Selesnjow, Zur Geschichte der Zeitung „Das freie Wort“, S. 955 f.

4 Weinert, Nationalkomitee, S. 69.

5 Übereinstimmend Petrick, „Freies Deutschland“, S. 62 ff. und Diesener, Propagandaarbeit, S. 11.

6 Weinert, Nationalkomitee, S. 78.

7 Diesener, Propagandaarbeit, S. 14.

8 Pütter, Rundfunk gegen das „Dritte Reich“, S. $280 \mathrm{ff}$.

9 Mayenburg, Hotel Lux, S. 320.

10 Geschäftsverteilungspläne des Instituts 99, RGASPI 578/5/4, Bl. 5 u. ebenda 495/77/39, Bl. 1; siehe Anlage Nr. 1; vgl. Leonhard, Revolution S. 285 ff. 
Staimer erweitert. Als Sprecher arbeiteten im Sender Friedrich Heilmann und - bevor er Redakteur wurde - Gustav von Wangenheim. Im Mai 1944 kam Wolfgang Leonhard als Sprecher hinzu. Der Sendeleiter war Bruno Schramm, der 1944 durch Lea Grosse und Egon Dreger Unterstützung bekam. Schreibkräfte waren zu Beginn Gerda Schering und Eleonore Staimer, die nach ihrem Wechsel auf einen Redakteursposten durch Vera Pisčassova ersetzt wurde ${ }^{11}$. Nach dem Kriegsende blieben in der Zeitungsredaktion lediglich Kurella, Bolz und Held, sowie als technische Mitarbeiterinnen Seiler und Gordeeva. Die Radioredaktion wurde mit Bernhard Gabelin, Inge von Wangenheim, Erna Haaf, Vera Pisčassova, Elisabeth Weinert und Lea Grosse faktisch neu besetzt ${ }^{12}$.

Um die Glaubwürdigkeit der Propaganda des NKFD zu erhöhen, insbesondere die der Zeitung, erschien von Januar 1944 bis Januar 1945 das „Freie Deutschland im Bild" in insgesamt zwölf Ausgaben ${ }^{13}$. Die Zeitung enthielt ausschließlich knapp untertitelte Bilder, die beweisen sollten, daß sich die als Propagandisten öffentlich auftretenden NKFD-Mitglieder gesund und lebendig in sowjetischer Gefangenschaft befanden. Die Illustrierte wurde von der "deutschen" Redakteurin Ruth von Mayenburg - einer Österreicherin - zusammengestellt. Mayenburg war zwar Angestellte des Instituts 99, arbeitete aber im Gebäude der Pravda, wo sie in den Redaktionsstab der vom GlavPURKKA herausgegebenen „Frontillustrierten“ eingegliedert wurde ${ }^{14}$.

Radio- und Zeitungsredaktion des Nationalkomitees hatten eine Dependance im Lager Lunevo. Kriegsgefangene Mitarbeiter der Zeitungsredaktion waren - laut eines Berichtes des UPVI - Major Homann, Leutnant Gerlach, Gefreiter Kerztscher, Generalmajor von Lenski, Oberleutnant von Kügelgen und Major Stößlein ${ }^{15}$. Abweichend von diesem UPVI-Bericht nennt das NKFD-Mitglied von Kügelgen in seinen Memoiren Wehrmachtspfarrer Kayser, Oberstleutnant Bredt, den Gefreiten Emendörfer und Major Hetz als Mitglieder des regelmäßig in Lunevo zusammenkommenden Redaktionskollegiums. Die Sitzungen seien von Rudolf Herrnstadt bzw. wegen dessen länger andauernden Krankheit von Lothar Bolz geleitet worden ${ }^{16}$. Die weitere Beteiligung Kriegsgefangener an der redaktionellen Arbeit des Nationalkomitees war breit gestreut. In der Zeitung schrieben - so eine nicht ganz vollständige Zählung - 274 unterschiedliche Autoren. Die meisten von ihnen lebten in den Offizierslagern Elabuga, Oranki, Suzdal' oder Voikovo bzw. in dem „antifaschistischen Renommierlager" Krasnogorsk ${ }^{17}$. Im Sender wurden ebenfalls beinahe alle Aktivisten an Übertragungen beteiligt: „Zwischen 1943 und 1945 kamen fast alle jene Mitglieder des NKFD wie des ,Bundes deutscher Offiziere über den Sender zu Wort, von denen angenommen werden konnte, daß sie durch ihren mili-

11 Ebenda; vgl. Leonhard, Revolution S. $303 \mathrm{ff}$.

12 Notizen Piecks über „die bei den Institutionen in Moskau verbleibenden Genossen“ vom Juni 1945, RGASPI 17/128/716, Bl. 70 (= SAPMO-BArch NY 4036/517, Bl. 155).

13 SAPMO-BArch, Bibliothek R-ZE 782 B 1943, 1944, 1945; vgl. Flugblätter des Nationalkomitees, S. 102; Weinert, Nationalkomitee, S. $76 f$.

14 Mayenburg, Blaues Blut und rote Fahnen, S. 345.

15 UPVI-Bericht über die Zeitungen für die deutschen Kriegsgefangenen, RGVA/K 1p/23a/9, Bl. 4.

16 Kügelgen, Nacht der Entscheidung, S. 467.

17 Petrick, „Freies Deutschland“, S. 61. 
tärischen Rang oder ihre menschlichen oder intellektuellen Fähigkeiten für Sendungen nach Deutschland oder an die Adresse der Wehrmacht von einiger propagandistischer Bedeutung waren. Insgesamt sollen über den Sender 179 Personen gesprochen haben." 18

Die Lenkung der Redaktionsarbeit führte, da die Kriegsgefangenen nicht das Gefühl haben sollten, bevormundet zu werden, zu einem komplizierten, im Endeffekt aber wirkungsvollen Abstimmungsverfahren. Dabei wurden die Themen und die Argumentationslinie der Autoren auf den Zivilsektorsitzungen im Institut 99 festgelegt und den kriegsgefangenen Redaktionsmitgliedern in Lunevo überbracht. Das UPVI berichtete darüber: „Die Thematik und die Argumentation wurden der Zweit-Redaktion vorgeschlagen, in Form von Anweisungen. Die Aufgabe der Vertreter der Haupt-Redaktion bestand darin, die Mitglieder der Zweit-Redaktion von der Richtigkeit des Plans für die nächste Ausgabe zu überzeugen und ihnen den Eindruck zu vermitteln, daß der Plan von ihnen ausgearbeitet worden sei, und ihnen die Richtigkeit der Thematik und der Argumentation zu beweisen."19 Aber auch der umgekehrte Weg war durchaus möglich. Vorschläge der Zweit-Redaktion wurden nach sorgfältiger Prüfung im Institut 99 ebenfalls angenommen. Allerdings wurden die aus Lunevo eingehenden Artikel in der Stadtredaktion gründlich überarbeitet. Diese Überarbeitung im Institut 99 wird faktisch zu gänzlich neuen Texten geführt haben, denn es sollen meist $50-75 \%$ eines Artikels umformuliert worden sein. Daher bestand die weitere Aufgabe der Redakteure vom Institut 99 darin, in einer zweiten Besprechung die kriegsgefangenen Autoren von der Richtigkeit der in der Stadtredaktion vorgenommenen Änderungen zu überzeugen. In der Mehrzahl der Fälle - so der Rechenschaftsbericht des UPVI - soll das auch gelungen sein ${ }^{20}$. Auch Kügelgen bestätigt: „Es herrscht ein eisernes Gesetz: Alles wird so lange und geduldig mit dem Autor diskutiert, bis er auch mit der kleinsten Änderung einverstanden ist. "21 Das gleiche Abstimmungsverfahren zwischen Stadt- und Lagerredaktion verwandte auch die Radioredaktion. Leonhard beschreibt die Zusammenarbeit der zivilen und der Kriegsgefangenen-Radioredaktion als kollegial und nahezu gleichberechtigt ${ }^{22}$. Während die Nachrichten und aktuellen Kommentare im Institut 99 produziert wurden ${ }^{23}$, fuhren vornehmlich von Wangenheim und Keilson nach Lunevo, um dort Aufrufe, Erklärungen und Kommentare aufzuzeichnen. Ab Juni 1944 wurden infolge der Gründung des kirchlichen Arbeitskreises des NKFD zusätzlich Sonntagspredigten für das Radioprogramm aufgenommen.

Die aufwendige Form der Redaktionsarbeit hatte innerhalb des Nationalkomitees den Effekt, daß sich die kriegsgefangenen Mitglieder nicht übervorteilt fühlten, was hinsichtlich ihrer Überzeugungskraft als Propagandisten keine geringe Rolle

18 Pütter, Rundfunk gegen das „Dritte Reich“, S. 282; dort auch eine namentliche Aufstellung der festen, sowohl zivilen als auch kriegsgefangenen Redaktionsmitglieder:

19 UPVI-Bericht über die Zeitungen für die deutschen Kriegsgefangenen, RGVA/K 1p/23a/9, Bl. 5.

20 Ebenda.

21 Kügelgen, Nacht der Entscheidung, S. 469.

22 „In jener Zeit, von 1943-45, legten alle Redakteure, vor allem auch Ackermann selbst, auf eine ehrliche, freundschaftliche Zusammenarbeit außerordentlichen Wert.", Leonhard, Revolution, S. $307 \mathrm{f}$.

23 Die Sendeanlagen befanden sich wieder an einem anderen Ort in Moskau - in der ulica Šablovka 34, ebenda, S. 302. 
gespielt haben dürfte. Für den Erfolg dieser Bemühungen spricht, daß es in der Erinnerungsliteratur keine Klagen über die Mitarbeit in den Redaktionen des Nationalkomitees gibt. Der nicht unerhebliche Aufwand war aber auch der Tatsache geschuldet, daß es sich aus sowjetischer Sicht bei der Zweit-Redaktion um feindliche Kriegsgefangene handelte, die in einem sowjetischen Propagandaorgan an die Weltöffentlichkeit traten. Denn berechtigterweise mußte die Sowjetunion davon ausgehen, daß jede Sendung und jede Nummer des „Freien Deutschland“ nicht nur von der deutschen Abwehr, sondern auch von den Alliierten genau verfolgt wurde ${ }^{24}$.

Der propagandistische Aufhänger für den Sender war die Übertragung von Grüßen aus der Kriegsgefangenschaft als Lebenszeichen für die Angehörigen ${ }^{25}$. Da das Deutsche Reich die Anwendung der Genfer Konvention gegenüber der Sowjetunion ablehnte, wurde die Annahme von Post verweigert, so daß die Familien im Ungewissen über das Schicksal der als vermißt gemeldeten Wehrmachtssoldaten waren. Die über den Sender ausgestrahlten Grüße trafen einen wunden Punkt der nationalsozialistischen Propaganda, die die Legende aufrechterhielt, die Rote Armee mache keine Gefangenen. Durch die Tätigkeit des Senders „Freies Deutschland" wurde die deutsche Seite zu Gegenreaktionen provoziert, die automatisch eine größere Öffentlichkeit für die NKFD-Propaganda schufen. Zum Beispiel sorgten die Radiosendungen mit dem NKFD-Mitglied Heinz Keßler, Sohn eines regional bekannten, kommunistischen Kommunalpolitikers und Gewerkschaftsfunktionärs in dessen Heimatstadt Chemnitz für Gerüchte und Unruhe, bis schließlich in den lokalen Zeitungen auf den Fall eingegangen wurde: „Zunächst hatte man die Familie unterrichtet, daß ich vermißt sei, möglicherweise gefallen oder gefangengenommen. Dies wurde auch öffentlich verbreitet, bis zu der Zeit, da ich mich an der Front durch Lautsprecheransprachen und Flugblätter, durch viele Aufrufe und Rundfunkerklärungen des Nationalkomitees deutlich bemerkbar machte. Von da an setzte, auch in den Chemnitzer Zeitungen, eine maßlose Welle der Verleumdung ein, gekrönt von der Nachricht, daß das Reichskriegsgericht mich in Abwesenheit zum Tode verurteilt hatte." 26

Stieß die Propaganda des NKFD im Falle der Familie Keßler auf ein politisiertes, tendenziell eher prosowjetisches Umfeld, so drang sie auch in die Wahrnehmungswelt vollkommen unpolitischer Menschen ein. Die damalige Ehefrau des NKFDMitgliedes Bernhard Bechler - eine dem NS-Regime loyal gegenüberstehende Offiziersfrau - wurde in anonymen Briefen von deutschen Radiohörern darüber informiert, daß sich ihr Mann lebend in russischer Gefangenschaft befinde ${ }^{27}$. Die Geschichte der Eheleute Bechler ist zudem ein extremes Beispiel für den Konflikt, in den sich die Propagandisten des NKFD begaben bzw. ihre Angehörigen brachten. Ein Kurier, der Margret Bechler Grüße von ihrem Mann übermittelte, wurde von

${ }^{24} \mathrm{Vgl.} \mathrm{zu}$ den westalliierten Reaktionen auf das NKFD Bungert, „Ein meisterhafter Schachzug", S. 93 ff.; dies., „Den deutschen Widerstandswillen brechen“, S. $52 \mathrm{ff}$.

25 Weinert, Nationalkomitee, S. 78.

${ }^{26}$ Keßler, Zur Sache und zur Person, S. 96; zu den nationalsozialistischen Gegenmaßnahmen siehe Heider, Reaktionen in der Wehrmacht, S. 614 ff.; Flugblätter des Nationalkomitees, S. $118 \mathrm{ff}$;; Das Nationalkomitee „Freies Deutschland“" und der Bund Deutscher Offiziere, S. $269 \mathrm{ff}$.

27 Bechler, Warten auf Antwort, S. $15 \mathrm{ff}$. 
der Gestapo verhaftet und später hingerichtet. Frau Bechler, die nicht wie die Familien anderer NKFD-Mitglieder in Sippenhaft genommen wurde, überstand mit Unterstützung der Nachbarn und Offizierskollegen ihres Mannes den Krieg, wurde aber im Juni 1945 vom NKVD verhaftetet und bis 1950 in sowjetischen Internierungslagern, dann bis 1956 in Gefängnissen der DDR festgehalten. Bernhard Bechler, der nach Kriegsende nach Deutschland zurückkehrte, trennte sich von seiner Frau und machte Karriere im neuen Regime in der SBZ/DDR 28.

Zeitung und Sender kommentierten regelmäßig die militärische Entwicklung an den Fronten und die politische Situation in Deutschland, wobei herausragenden Ereignissen viel Platz eingeräumt wurde. Eine wesentliche Aufgabe war die Verbreitung einschlägiger alliierter Verlautbarungen sowie der Aufrufe von NKFD und BDO. In seiner Propaganda argumentierte das NKFD von Anfang an damit, daß der Krieg verloren sei und Deutschland sich, sofern sich kein Widerstand gegen Hitler rege, den Forderungen der fest zusammenstehenden Alliierten beugen müsse. Im Sommer 1944 standen daher im Mittelpunkt der Berichterstattung die Eröffnung der zweiten Front, der Zusammenbruch der Heeresgruppe Mitte und das Attentat auf Hitler. Diese Ereignisse wurden als Bestätigung der vom NKFD vorausgesagten deutschen Niederlage gewertet und in der Propaganda entsprechend dargestellt. Nach Kriegsende konzentrierten sich die Redaktionen auf die Frage der Wiedergutmachung. Die Auseinandersetzung mit dem Nationalsozialismus wurde verbunden mit der Aufforderung an die Kriegsgefangenen, durch gute Arbeit in der UdSSR Wiedergutmachung zu leisten. Zusätzlich sollte durch die Darstellung der Ereignisse in der sowjetischen Besatzungszone die friedliche Politik der Sowjetunion hervorgehoben werden. Birgit Petrick kommt bei ihrer Auswertung der Beiträge des „Freien Deutschland“ zu deutschlandpolitischen Themen zu dem Fazit: „Die im Freien Deutschland niedergelegten Vorstellungen des NKFD/ BDO zur Neugestaltung Deutschlands werden einerseits aus der Negation des in Deutschland Bestehenden, andererseits im Hinblick auf die Sowjetunion - in beispielgebender Funktion - konzeptualisiert. Sie müssen vor dem Hintergrund der politischen und militärischen Ereignisse gewertet werden."29

Die Redaktionen im Institut 99 hatten dafür zu sorgen, daß die Propaganda im Namen des Nationalkomitees mit den alliierten Zielen in Einklang zu bringen war. Als Kontrollinstanz gab es eine direkte Zensur durch die Politische Hauptverwaltung. Für die Zeitung fungierte als Zensor der ungarische Kommunist und Mitarbeiter der 7. Verwaltung Ernö Gerö. Die Arbeit des Senders überwachte der stellvertretende Leiter der 7. Verwaltung Oberst Braginskij, der gelegentlich durch die

28 Papadopoulus-Kilius, „Es gibt zwei Deutschlands ...“, S. 216. Zur Sippenhaft für Angehörige des NKFD siehe Ueberschär, Das NKFD und der BDO im Kampf gegen Hitler, S. 39 f; Heider, Reaktionen in der Wehrmacht, S. 631 f.; Wegner-Korfes, Weimar - Stalingrad - Berlin, S. $182 \mathrm{ff}$.

29 Petrick, „Freies Deutschland“, S. 193. Im weiteren irrt Petrick: „So war nach der Moskauer Außenministerkonferenz im Oktober 1943 die Annahme nicht mehr realistisch, daß sich die Sowjets des NKFD/BDO bedienen würden, um zu einem Separatfrieden mit Deutschland zu kommen. Damit verschlechterten sich auch die Aussichten des NKFD/BDO, Kern einer neuen deutschen Regierung zu werden" (ebenda). Antworten auf Fragen der sowjetischen Deutschlandpolitik können nicht aus der Analyse der Propaganda des NKFD gewonnen werden. Die internen Diskussionen um die Frage einer deutschen Exilregierung waren mitnichten im Oktober 1943 abgeschlossen. 
deutsche Kommunistin Frida Rubiner vertreten wurde ${ }^{30}$. Bei politisch brisanten Themen wurde die Redaktionsarbeit unmittelbar von der Leitung der Politischen Hauptverwaltung kontrolliert. Dies erfolgte durch das direkte Eingreifen Manuil'skijs, der sich dabei bei Ščerbakov rückversicherte ${ }^{31}$.

Durch die Tätigkeit des Instituts 99, d.h. die Instruktion auf den Zivilsektorsitzungen im allgemeinen und die Arbeit der Stadtredaktionen im besonderen, gelang es, die Propaganda des NKFD der außenpolitischen Position der Sowjetunion anzugleichen und gleichzeitig die einzelnen Mitglieder des Nationalkomitees in dem Glauben zu wiegen, sie agierten weitgehend frei.

\section{Lagerbevollmächtigte des Nationalkomitees}

Lagerbevollmächtigte des NKFD wurden trotz anders lautender Beschlußfassung ab Januar 1944 eingesetzt. Obwohl das Plenum des Nationalkomitees bereits auf seiner ersten Vollsitzung am 14. Juli 1943 die Entsendung von Bevollmächtigten in die Lager beschlossen hatte, befahl erst die Direktive des UPVI vom 23. Dezember 1943 den Lagerverwaltungen die Auswahl von geeigneten Lagerbevollmächtigten ${ }^{32}$. Die ersten 25 Lagerbevollmächtigten des NKFD wurden im Januar 1944, weitere 27 im Februar ernannt ${ }^{33}$. Bereits die verzögerte Umsetzung des NKFD-Beschlusses deutet darauf hin, daß das NKFD bzw. das Institut 99 nicht allein entscheiden konnte, sondern bei der Umsetzung auf den Apparat des UPVI vor Ort angewiesen war.

Die Nominierungen der Lagerbevollmächtigten wurden auf Sitzungen des sogenannten Lager-Aktivs durchgeführt. So berichtete die Politleitung des Lagers Spaso-Zavodsk (Nr. 99) unter Bezug auf die Direktive des UPVI, daß eine „Besprechung mit dem engsten Kreis des antifaschistischen Aktivs (Absolventen der Antifa-Schule)“ sowie "eine Versammlung des antifaschistischen Aktivs deutscher Kriegsgefangener zur Beratung der Kandidatur eines Bevollmächtigten“ durchgeführt worden seien ${ }^{34}$. Die Personalunterlagen des vorgeschlagenen Kandidaten wurden zusammen mit denen weiterer drei Kandidaten aus anderen Lagern von der Politabteilung des UPVI an Manuil'skij, d.h. den Rat für militärpolitische Propaganda geschickt. Kurz darauf folgten die Unterlagen weiterer 14 Kandidaten ${ }^{35}$. Diese Unterlagen bestanden aus der von der Politabteilung des Lagers ausgestellten Charakteristik des Kandidaten und dem vom Kriegsgefangenen selbst verfaßten Lebenslauf („Autobiographie“), der die vollzogene Wandlung zum Antifaschisten be-

30 Leonhard, Revolution, S. 290 ff. und S. 309; zu Gerö siehe Borsányi, Ernö Gerö, S. 275 ff.

31 Leonhard, Revolution, S. 292.

32 Arbeitsbericht der Politabteilung des UPVI vom 31. 12. 1943, RGVA/K 1p/9a/7, Bl. 88.

33 Vgl. Tätigkeitsbericht der Politabteilung des UPVI vom 11.2. 1944, RGVA/K 1p/11a/1, Bl. 47; Rapport über die politische Arbeit des UPVI im Januar/Februar 1944, ebenda Akte 2, Bl. 14.

34 Undatierter Bericht der Lagerleitung des Lagers Nr. 99 an UPVI-Chef Petrov [vermutlich Anfang Januar 1944], RGVA/K 4p/4/31, Bl. 54. Die meisten der im folgenden zitierten Berichte von den Lagerleitungen nehmen Bezug auf die UPVI-Direktive Nr. 28/p/21378 vom 23. 12.1943.

35 Jakovec an Manuil'skij am 16. 1.1944 und 3. 2. 1944, RGVA/K 4p/4/31, Bl. 149-150. 
legen sollte. In einer vom Politinstrukteur und der Leitung des Lagers unterschriebenen "Auskunft" (spravka) wurden alle für die Kaderentscheidung relevanten Punkte noch einmal zusammengefaßt: Schul- und Berufsbildung, militärische Laufbahn bis zur Gefangennahme, politischer Hintergrund des Kriegsgefangenen und eventuell auch der der engsten Familienangehörigen, Engagement im Lageraktiv und gegebenenfalls die Teilnahme an antifaschistischen Schulungen.

Bei der politischen Beurteilung eines Kriegsgefangenen spielte der Politinstrukteur die maßgebliche Rolle. Häufig war er bereits bei der ersten Vernehmung eines neu aufgenommenen Gefangenen anwesend und konnte dabei eine erste Einschätzung vornehmen. Diese begleitete den Kriegsgefangenen auf seinem weiteren Weg als Objekt der politischen Arbeit im Lager. Bei der Aufnahme mußte außerdem jeder Kriegsgefangene einen Fragebogen ausfüllen, dem später die personenbezogenen Angaben entnommen werden konnten ${ }^{36}$. Über die Tätigkeit der Politinstrukteure, in der Regel deutsche Exilkommunisten, ging das Interesse der KPD, kommunistisch eingestellte Kriegsgefangene zu rekrutieren und in die Arbeit der Partei einzubinden, mit dem Interesse des UPVI, Lager-Aktivs zu initiieren, Hand in Hand $^{37}$. So dominierte bei der Auswahl der NKFD-Bevollmächtigten im Lager Novosibirsk (Nr. 199/Zone I-VI) im Januar 1945 der Typus des kommunistisch organisierten Soldaten mit proletarischem Hintergrund, der freiwillig zur Roten Armee übergelaufen war. Alle vorgeschlagenen Kandidaten waren Mannschaftsdienstgrade und kamen aus Arbeiter- bzw. Bauernfamilien. Sie hatten zumeist acht Jahre Volksschulbildung und waren Arbeiter oder Handwerker. Angaben, die von den Kriegsgefangenen über ihre KPD/KJVD-Mitgliedschaft gemacht wurden, konnten von den Politinstrukteuren nicht überprüft werden und galten daher nur unter Vorbehalt, aber sie waren offenbar ein Auswahlkriterium. So hatten von den sechs NKFD-Bevollmächtigten im Lager Nr. 199 zwar drei die Antifa-Schule nicht besucht, jedoch konnten von diesen zwei auf eine KPD-Mitgliedschaft verweisen. Damit hatten fünf von sechs ausgewählten Bevollmächtigten entweder die AntifaSchule absolviert oder waren kommunistisch organisiert gewesen ${ }^{38}$.

An einem weiteren Beispiel wird deutlich, daß der Besuch der Antifa-Schule das gewichtigste Kriterium war. Im weißrussischen Lager Bobrujsko (Nr. 56) wurden als Bevollmächtigte des NKFD, des BDO und des Büros österreichischer Antifaschisten drei Offiziere mit akademischer Ausbildung vorgeschlagen, von denen einer Berufssoldat, ein anderer Mitglied der NSDAP war. Für Oberleutnant Dr. Horst H., Amtsgerichtsrat aus Plauen und Mitglied der NSDAP seit 1933, am 2. 2. 1943 in Stalingrad in Gefangenschaft gegangen und von Mai bis November 1944 die Antifa-Schule besucht, lautete die Charakteristik: „Anfang Dezember 1944 kam er in das Lager Nr. 56, wo er im Aktiv der Antifaschisten der Zone I des Lagers arbei-

36 Seitens des UPVI wurde aus diesen Materialien zu jedem Gefangenen eine „Registrier-Akte“ (učetnoe delo) angelegt. Im Unterschied dazu gab es auch Personalakten (ličnoe delo), in der personenbezogene Angaben zu „Bewährungen“ (z. B. Einsatz als Lageraktivist), zur „operativen Verwendung" (z.B. Spitzeldienste im Lager) oder auch über eventuell vorhandenes „kompromittierendes Material“ (z. B. Verstrickung in Kriegsverbrechen) gesammelt wurde; vgl. Karner, Archipel GUPVI, S. 65 f.; Robel, Antifa, S. 33 f.

37 Vgl. Robel, Antifa, S. 35.

38 Brief der Leitung des Lagers Nr. 199 an UPVI-Chef Petrov vom 25. 1. 1945 mit den Charakteristiken in der Anlage, RGVA/K 4p/5/19, Bl. 70-76. 
tete. In der Lagerzone führt $\mathrm{H}$. eine eigenständige propagandistische Arbeit unter den Kriegsgefangenen durch, hält Vorlesungen und Vorträge, führt Gespräche über politisch-antifaschistische Themen, nimmt aktiv an der gesamten im Lager durchgeführten antifaschistischen Arbeit teil und genießt eine große Autorität unter den Antifaschisten und unter allen Kriegsgefangenen. $\mathrm{H}$. ist diszipliniert, ausnehmend fleißig und eifrig. In der Zeit seines Aufenthaltes im Lager hat er sich von einer positiven Seite gezeigt. Er ist entwickelt und ist in der Lage erzieherische Arbeit zu leisten. Seitens des Lagers Nr. 56 wird seine Bestätigung als Bevollmächtigter des Nationalkomitees ,Freies Deutschland" befürwortet. " 39 Es scheint demnach, daß in Mannschaftslagern vorwiegend kommunistisch eingestellte Soldaten, bevorzugt KPD-Mitglieder und Überläufer, als Bevollmächtigte eingesetzt wurden. In den Offizierslagern dagegen mußten die Kandidaten zumindest die Antifa-Schule absolviert haben, um die in der Regel bürgerliche Herkunft und ihr unter Umständen systemkonformes Verhalten im nationalsozialistischen Deutschland (Mitgliedschaft in der NSDAP) durch die „antifaschistische Erziehung“ zu kompensieren.

Die sehr unterschiedliche Kaderauswahl findet eine weitere Erklärung darin, daß die Ernennung von Lagerbevollmächtigten zwar auf Vorschlag des UPVI (Politinstrukteur und Lagerverwaltung) erfolgte, die Entscheidung aber beim Institut 99 lag. Dadurch entstand eine doppelte Kaderverwaltung. Das UPVI gab zwar die Unterlagen zur Entscheidung an das Institut 99 weiter, fertigte für die eigene Verwaltung jedoch Kopien an und ließ die von Kriegsgefangenen in ihren Autobiographien gemachten Angaben von der operativen Abteilung des UPVI überprüfen. Aus Sicht des UPVI stellte sich daher die Ernennung eines Lagerbevollmächtigten beispielhaft folgendermaßen dar: „Auf der Grundlage Ihrer Direktive Nr. 28/p/21378 vom 23. Dezember 1943 ist im NKVD-Lager Nr. 84 der Bevollmächtigte des Nationalkomitees ,Freies Deutschland' G. Theo Theodorovič ausgewählt worden. Die Kandidatur G.s ist im antifaschistischen Aktiv mit ausschließlich positiver Resonanz beraten worden. Die Kandidatur entspricht [unseren Vorstellungen]. Ich bitte um Bestätigung." 40 Die Bestätigung erfolgte durch UPVIChef Petrov, der eine Kopie behielt und das Original an Manuil'skij weiterreichte ${ }^{41}$. Bei neuerlichen Nominierungen von Lagerbevollmächtigten des NKFD befinden sich auf den Meldungen an das UPVI regelmäßig die Eingangsstempel der operativen Abteilung sowie beispielsweise die Anweisung Petrovs „Ordnen Sie die Charakteristiken über die Bevollmächtigten in die bereits vorhandenen ein"42. Das UPVI war nicht gewillt, jegliche Kontrolle an den Rat für militärpolitische Propaganda bzw. das Institut 99 abzugeben. Da aber die Originale der Personalunterlagen an das Institut 99 gingen, war dessen Kaderverwaltung bis zur Auflösung des

39 Charakteristik der Lagerleitung eines Kandidaten für das Amt des NKFD-Lagerbevollmächtigten, unterschrieben vom stellvertretenden Leiter des Lagers Nr. 56 und dem Instrukteur der Politabteilung der Lagerverwaltung, RGVA/K 4p/5/19, Bl. 41; ebenda Bl. 45+RS und Bl. 49 die Charakteristiken der anderen beiden Bevollmächtigten.

40 Chef des Lagers Nr: 84 an UPVI-Chef Petrov am 13.1. 1944 mit der Charakteristik als Anlage, RGVA/K 4p/4/31, Bl. 11-12.

41 Ebenda; Petrov machte auf dem Brief den Vermerk: „Eine Kopie der Charakteristik für uns anfertigen und das Original an Manuil'skij".

42 Schreiben der Leitung des Lagers Nr. 182 an Petrov vom 10. 2. 1945, RGVA/K 4p/5/19, B1. 1; in derselben Akte befinden sich mehrere derartige Schreiben. 
Instituts Ende 1946 die entscheidende Instanz ${ }^{43}$. Und im Gegensatz zum UPVI konnte das Institut 99 bei der Einsetzung von Lagerbevollmächtigten auf die Absolventen der Antifa-Schulen zurückgreifen, deren Kaderverwaltung ebenfalls vom Institut 99 geführt wurde. Auf diese Weise gab es zwei Wege der Kaderrekrutierung.

Der tatsächliche Einfluß des Instituts 99 auf die unmittelbare politische Arbeit in den Lagern blieb gering. Zwar gab das Nationalkomitee „Mitteilungen für die Lagergruppen" heraus, aber vermutlich erschienen im ganzen nur zwei Nummern ${ }^{44}$. Der Effekt der Mitteilungen in den Lagern war wohl verschwindend gering. So wurden in der ersten Ausgabe die Aufgaben der Lagergruppe des NKFD beschrieben, die sich jedoch nicht von denen der bereits bestehenden Lager-Aktivs unterschieden: „Der Lagerausschuß hat die Bewegung im Lager zu organisieren, die politische Arbeit nach den Richtlinien des Nationalkomitees zu leiten, dem Nationalkomitee monatlich zu berichten. (...) Eine besondere Aufgabe des Lagerausschusses ist die listenmäßige, organisatorische Zusammenfassung und Schulung derjenigen Mitglieder der Lagergruppe, die sich zu aktiven Kämpfern der Bewegung ,Freies Deutschland' eignen (zu Rednern, Organisatoren, Mitarbeitern für Zeitung, Flugschriften, Flugblätter usw.). Er hat bei der Lagerverwaltung zu erwirken, daß alle wichtigen Funktionen der Lagerselbstverwaltung (Lager- und Barackenältester, Brigadeführer, Küchenverwaltung, Sanitätspersonal) von anständigen Männern besetzt werden, die keine nationalsozialistische Gegenpropaganda treiben." 45 Damit wurde in den Mitteilungen lediglich der Status quo beschrieben, der auch schon vor Gründung des NKFD bestanden hatte.

Die Einwirkung auf die Kriegsgefangenen erfolgte durch Formen, die seit dem Aufbau der Lager-Aktivs im Herbst 1941 eingeübt waren. Diese waren für die Ziele des Nationalkomitees durchaus kontraproduktiv. Durch die bevorzugte Besetzung der Funktionsstellen mit Mitgliedern des Aktivs, egal ob es sich nun „Antifa-Aktiv“ oder "Lagergruppe des NKFD“ nannte, bildete sich die sogenannte Lagerprominenz, die die begehrten, mit Besserstellung verbundenen Posten in der Selbstverwaltung des Lagers unter sich verteilte. Dieser Mechanismus wurde vom UPVI instrumentalisiert ${ }^{46}$. Mit Hinweis auf den organisatorischen Nutzen für die Lagerverwaltung wurden die Politinstrukteure ausdrücklich angewiesen, die Aktivisten zu bevorzugen: „Es ist zweckmäßig, als Älteste der Hundertschaften solche Antifaschisten zu bestimmen, die das Vertrauen ihrer Kameraden haben. Sie sollen zusätz-

43 Archivtechnisch aufschlußreich ist, daß nach Auflösung des Instituts 99 alle Kaderunterlagen über die Kriegsgefangenen im UPVI-Archiv (heute eine Abteilung des RGVA/K) abgelegt wurden. Die dort im Bestand „fond 88“ abgelegten Akten tragen die Aufschrift „Institut $99^{\circ}$. Leider ist der Bestand gesperrt.

44 Wahrscheinlich wurden die Mitteilungen nach der zweiten Nummer (SAPMO-BArch NY 4009/28, Bl. 217-242) eingestellt. Weinert (Nationalkomitee, S. 66 f.) zitiert in seinem Bericht lediglich das Inhaltsverzeichnis der zweiten Ausgabe und kommt im weiteren nicht mehr auf sie zu sprechen. Vgl. Zirke, Im Tosen des Krieges geschrieben, S. $111 \mathrm{f}$.

45 „Mitteilungen des Nationalkomitees ,Freies Deutschland“ für die Lagergruppen Nr. 1“, SAPMO-BArch NY 4091/111 Ü, B1. 26-27.

46 Frieser (Krieg hinter Stacheldraht, S. $144 \mathrm{ff}$.) nennt das „System von Gratifikation und Pression": Durch die Belohnung des Bekenntnisses zur Antifa einerseits und die Bestrafung politischer Opposition andererseits sei das Lagerleben zu einem ideologischen und zudem physischen Existenzkampf geworden. 
liche Lebensmittel erhalten. Die Schaffung eines möglichst großen geschulten antifaschistischen Aktivs wird auch dazu beitragen, die Lager-Disziplin zu festigen." 47 Und ein Rundbrief des UPVI vom September 1943 legte den Lagerchefs nahe: „Verschaffen Sie den Absolventen der [Antifa-]Schule auch die materiellen Voraussetzungen. Gleichen Sie die Verpflegung der Arbeiternorm an. Sorgen Sie dafür, daß sie Kleidung und Schuhe haben. Sie müssen spüren, daß das Verhalten ihnen gegenüber etwas anderes ist als zu den übrigen Kriegsgefangenen. Lassen Sie auch nicht diejenigen [Antifa-Absolventen] aus den Augen, die als Brigadiere und gewöhnliche Arbeiter geschickt werden. " 48 Der Effekt bei den politisch inaktiven Kriegsgefangenen war genau entgegengesetzt. Die Antifa-Prominenz im Lager war verhaßt. Diejenigen, die nur irgendwie den Aktivitäten der Lagergruppe Interesse oder Neugier entgegenbrachten, wurden sofort von ihren Mitgefangenen geschnitten. Zaghafte Annäherungsversuche wurden seitens der Aktivs auch dadurch erschwert, daß in der Wandzeitung sofort darüber berichtet wurde. Sobald aber der Name eines bis dahin politisch nicht aktiven Gefangenen für das ganze Lager mit der Antifa in Verbindung gebracht werden konnte, brachen alle seine sozialen Kontakte zusammen. Auch wenn es unter den Offizieren offenbar möglich war, durch eine Art Ehrengericht wieder in den Kreis der Kameraden aufgenommen zu werden, ergab sich für den vereinzelten Kriegsgefangenen in jedem Fall die Zwangslage, ein (politisches) Bekenntnis für die eine oder die andere Seite abgeben zu müssen ${ }^{49}$. Die Mitglieder des Lager-Aktivs werden daher auch als eine „am sozialen Rande zwischen deutschen Kameraden und sowjetischer Gewahrsamsmacht" stehende Gruppe charakterisiert ${ }^{50}$.

Die Erfolge der politischen Arbeit in den einzelnen Lagern waren gering. In den Berichten der Lagerverwaltungen sollte das kaschiert werden, so daß im April 1944 die UPVI-Leitung anmahnte:,In den monatlichen Berichten fast aller Lager kommt die Arbeit der Antifaschisten, die eine Antifa-Schule absolviert haben, überhaupt nicht zum Ausdruck. Die Zahl der Antifaschisten und der Aktivs wird nicht mitgeteilt. In den Politmitteilungen wird lediglich die monatliche Zunahme der Zahl der Antifaschisten genannt, aber wieviele Antifaschisten es insgesamt geworden sind, ist aus den Berichten nicht ersichtlich. Auf die Arbeit der Bevollmächtigten des Nationalkomitees wird nicht näher eingegangen. Ein wesentlicher Indikator für diese Arbeit - die Zahl derjenigen, die sich der Plattform des Nationalkomitees angeschlossen haben - fehlt in den Berichten. "51 Bis Kriegsende hatte die politische Arbeit des NKFD in den Lagern lediglich fragmentarischen Charakter. In längst nicht allen Lagern wurden die Ziele des NKFD durch eigens berufene Bevollmächtigte verbreitet. In der Regel stützten sich die Lagerleitungen auf die wenigen kooperationswilligen, meist kommunistischen Aktivisten. Einen Einblick in die politische Arbeit in einem gewöhnlichen Arbeitslager für Kriegsgefangene gibt der Inspektionsbe-

47 EKKI-Richtlinien vom 24. 1. 1942, RGASPI 495/77/30, Bl. 13.

48 UPVI-Rundbrief an die Lagerchefs vom 29. 9. 1943, RGVA/K 4p/3/1, Bl. 116+RS.

49 Vgl. die Schilderung bei Kügelgen (Nacht der Entscheidung, S. 324 ff.), in der dieser, obwohl er mehr die Darstellung einer „Läuterung zum Antifaschisten“ intendiert, detailliert alle hier nur skizzierten Vorgänge beschreibt.

50 Lehmann, Gefangenschaft und Heimkehr, S. 50.

51 Rundbrief der UPVI-Leitung an die Lagerverwaltungen vom 15.4. 1944, RGVA/K $1 \mathrm{p} / 33 \mathrm{a} / 16, \mathrm{Bl} .187$. 
richt Herbert Hentschkes, der im Februar und März 1945 neun Außenlager des ukrainischen Lagers Nr. 256 besuchte. Der Arbeitsbetrieb - Bergbau - ließ eine kontinuierliche politische Arbeit nicht $\mathrm{zu}$, da die Kriegsgefangenen rund um die Uhr in Schichten arbeiteten. Es gab zwar Lager-Aktivs, aber weder im Stamm- noch in den Außenlagern war ein Politinstrukteur eingesetzt. Schulungsmaterial war nicht vorhanden. Die Propaganda des Nationalkomitees hatte überhaupt keine Berücksichtigung gefunden: „Meistens gingen die Aktive (vor allem auch die sich dort befindlichen Kommunisten) von der Perspektive der proletarischen Revolution nach Beendigung dieses Krieges aus, und sie waren bestrebt, anstatt einer breiten Lagergruppe der Bewegung Freies Deutschland, wenn auch nicht direkt eine kommunistische Parteiorganisation, so doch immerhin eine kommunistische Organisation zu schaffen." 52

Die unmittelbare Arbeit des NKFD und damit der Einfluß des Instituts 99 beschränkte sich auf einige wenige Kriegsgefangenenlager, streng genommen nur auf die Offizierslager. Nach der Gründung des Nationalkomitees im Juli 1943 waren Delegationen in die Offizierslager Krasnogorsk (Nr. 27), Elabuga (Nr. 97), Oranki (Nr. 74) und Suzdal' (Nr. 160) sowie in das Generalslager Voikovo (Nr. 48) gefahren, um für den zu gründenden Offiziersbund zu werben. Immerhin unterschrieben den Gründungsaufruf des BDO 95 Offiziere, aber die weitere Werbung blieb, wie der Zivilsektor im Institut 99 im April 1944 urteilte, „schwach“53. Der Grund lag offensichtlich darin, daß es entgegen des ursprünglichen Beschlusses des NKFD über die Entsendung von Lagerbevollmächtigten bei zeitweiligen Delegationsreisen geblieben war. Ständige Bevollmächtigte wurden erst ab Januar 1944 eingesetzt, das aber nur in den Mannschaftslagern, in denen es ein ohnehin funktionierendes (kommunistisches) Antifa-Aktiv gab. In den Offizierslagern waren neben dem Lager Krasnogorsk faktisch nur in Elabuga Bevollmächtigte ständig eingesetzt. Dabei war die Einbeziehung dieses Lagers vom Institut 99 auch erst im April 1944 beschlossen worden ${ }^{54}$.

Möglichkeiten, die Tätigkeit in den Offizierslagern auszuweiten, ergaben sich im Sommer 1944 durch die sprunghaft angestiegene Zahl deutscher Kriegsgefangener, vor allem durch den Zustrom zahlreicher Generäle und Stabsoffiziere der zusammengebrochenen Heeresgruppe Mitte ${ }^{55}$. Anfang Juli 1944 fuhr eine siebenköpfige BDO-Delegation in das Lager Grjazovec (Nr. 150), in das die Mehrzahl der gefangengenommenen Offiziere gekommen war. Während Major von Frankenberg und Proschlitz und Hauptmann Burmeister - quasi als Lagerbevollmächtigte - bis Dezember 1944 im Lager blieben, fuhren die restlichen Delegationsmitglieder Korfes, Hetz und Hadermann nach einigen Tagen wieder $\mathrm{ab}^{56}$. Gleichzeitig hielten sich Major Stößlein und Wehrmachtspfarrer Kayser in Begleitung von Paul Försterling vom

52 Bericht Herbert Hentschkes vom 21. 3. 1945, SAPMO-BArch NY 4036/572, Bl. 28.

53 Beschlußprotokoll des Instituts 99 vom 10. 4. 1944, RGASPI 495/77/46, Bl. 2; vgl. die Notizen Piecks zu dieser Sitzung, SAPMO-BArch NY 4036/499, B1. 100.

54 Ebenda.

55 Beschlußprotokoll des Instituts 99 vom 14. 8. 1944, RGASPI 495/77/46, Bl. 11; siehe auch Frieser, Krieg hinter Stacheldraht, S. $244 \mathrm{ff}$.

56 Notizen Korfes, BArch Abt. Potsdam 90 KO 10/29, Bl. 1-3 (Die ursprüngliche Reihenfolge ist entgegen der Archivpaginierung: Bl. 3 RS, 3, 2, 2 RS, 1, 1 RS); vgl. Frankenberg, Meine Entscheidung, S. $244 \mathrm{ff}$. 
11. Juli bis zum 23. August 1944 im Offizierslager Elabuga auf. Zusammen mit dieser NKFD-Delegation fuhr eine Gruppe des BDO bestehend aus General Schlömer, General Lattmann und Leutnant Einsiedel ebenfalls nach Elabuga ${ }^{57}$. Nach der Rückkehr aus dem Offizierslager berichtete Försterling der KPD-Führung, daß das Auftreten der BDO-Mitglieder auf Widerspruch und Ablehnung gestoßen sei. Allgemein bemängelte er die personell schwache Besetzung des LagerAktivs und die „ideologisch schwankende Haltung der einfachen und auch der leitenden Alktivisten, z. B. in der Polenfrage [Frage der polnischen Westgrenze]". Bevor die Delegation das Lager Elabuga verlassen habe, sei „im allgemeinen Einverständnis mit der Lagerleitung und den Aktivisten in den verschiedenen Lagern ein ständiger Ausschuß gebildet" worden ${ }^{58}$. Jedoch wurde die Einsetzung dieses Lagerbevollmächtigten vom Institut 99 rückgängig gemacht, was abermals auf dessen Anspruch hinweist, Kaderentscheidungen nicht abgeben zu wollen. Zur Umsetzung jedoch - das zeigt der Beschluß des Instituts 99 ebenfalls - war man auf die Lagerverwaltung des UPVI, insbesondere deren operative Abteilung, angewiesen: „Die Frage der Ablösung des Bevollmächtigten P. soll durch die zuständigen Organe geregelt werden." 59

Aufgrund der Quellenlage fällt es nach wie vor schwer, einen Überblick über die Wirksamkeit des Nationalkomitees in allen Kriegsgefangenenlagern zu bekommen. Die Tätigkeit des BDO ist insofern gut zu beschreiben, als sie sich auf die wenigen Offizierslager beschränkt. Aufgrund der propagandistischen Wirkung, die durch die Gewinnung von Offizieren oder Generälen erzielt werden konnte, hatte sie immer exklusiven Charakter. Nach zwei Monaten Werbung im Lager Grjazovec waren bis September 1944402 neue BDO-Mitglieder gewonnen worden. Zum 1. November 1944 sollen es 1100 Neuzugänge gewesen sein 60 . Insgesamt registrierte eine interne BDO-Aufzeichnung zum 1. September 19441316 BDO-Mitglieder61. Demnach waren bis zum Frühjahr 1944 nur ungefähr 200 Offiziere dem BDO beigetreten; davon 95 bei der Gründung des Offiziersbundes. Der größte $\mathrm{Zu}-$ wachs an Mitgliedern muß um die Jahreswende 1944/45 gekommen sein. Eine leider nicht datierte Aufstellung Ackermanns beziffert die Zahl der BDO-Mitglieder auf „rund 4000“, von denen 7-8\% Stabsoffiziere gewesen seien ${ }^{62}$. Auf den Gesamtzeitraum der Tätigkeit von NKFD und BDO bezogen wird daher Puttkamers Ein-

57 Einsiedel, Tagebuch der Versuchung, S. $125 \mathrm{ff}$.

58 Delegationsbericht Försterlings vom 31. 8. 1944, SAPMO-BArch NY 4036/572, Bl. 36 und Bl. 37 RS; zum Widerstand gegen das Auftreten der NKFD/BDO-Delegation in Grjazovec siehe Hahn, Ich spreche die Wahrheit, S. 153.

59 Beschlußprotokoll des Instituts 99 vom 18. 9. 1944, RGASPI 495/77/46, Bl. 21.

60 Frankenberg, Meine Entscheidung, S. $244 \mathrm{ff}$.

61 „Notizen zu Entstehung und Aufgaben des BDO“, BArch Abt. Potsdam 90 KO 10/28, B1. 29.

62 „Der Bund deutscher Offiziere zählt gegenwärtig rund 4000 Mitglieder, davon 1 Generalfeldmarschall, 51 Generäle, ctwa 40 Oberste, etwa 50 Oberstleutnants, etwa 150 Majore, etwa 400 Hauptleute, die übrigen [ca. 3300] Oberleutnants und Leutnants.“, „Das Nationalkomitee Freies Deutschland und der Widerhall auf seine Tätigkeit", ohne Datum Vvermutlich Frübjabr 1945], handschriftlicher Vermerk „von Ackermann“, SAPMO-BArch NY 4036/580, Bl. 50. Vgl. Lexikon der Parteigeschichte, S. 302, das diese Angabe auf „Anfang 1945 “ datiert. Ebenda werden bis zur Gründung des BDO (12.9. 1943) 100 Offiziere als Mitglieder des NKFD gezählt. 
schätzung zutreffend sein: „Außer dem Lager 97 [Elabuga] mögen es noch 4 oder 5 andere gewesen sein, in die Delegationen entsandt wurden. So konnte in Wirklichkeit von einer Führung durch den Lunower ,Wasserkopf' keine Rede sein."63

Bei der Auflösung des NKFD räumte Komiteepräsident Weinert ein, daß es „nicht sehr viele Meldungen aus den Lagern“ gegeben habe und daß diese nur durch die Politinstrukteure zustandegekommen seien ${ }^{64}$. Ebenso waren die Einwirkungsmöglichkeiten des NKFD auf die politische Arbeit in den Lagern sehr gering geblieben. Nach Auflösung des Nationalkomitees berichtete Weinert an Wilhelm Pieck: „In der Zeitung [Freies Deutschland] wurden die Bedürfnisse und Aufgaben der Gefangenenlager in den Vordergrund gestellt. Diese Aufgabe wurde sehr dadurch erschwert, daß so gut wie keine Verbindung mit den Lagern besteht und auch trotz aller Anträge nicht geschaffen wurde."65 Demnach hatte sich durch die Gründung des Instituts 99 nichts an der politischen Arbeit in den Kriegsgefangenenlagern geändert.

Der NKFD-Beschluß über die Einsetzung von Lagerbevollmächtigten bekam erst durch die entsprechende UPVI-Direktive Wirkungskraft, deren Umsetzung vom Institut 99 kaum beeinflußt werden konnte und in erster Linie von den Politinstrukteuren in den Lagern abhing. Diese behielten die Arbeitsformen bei, wie sie bereits vor der Gründung des Nationalkomitees angewandt worden waren ${ }^{66}$. Insbesondere bei der politischen Arbeit in den Offizierslagern wurde seitens des UPVI offenbar darauf geachtet, möglichst keine festen Bevollmächtigten des BDO zuzulassen, sondern es bei zeitlich befristeten Delegationsreisen zu belassen. Für die Lagerbevollmächtigten des NKFD in den Mannschaftslagern mag das etwas anderes gewesen sein, da sie vorwiegend ohnehin aus dem Kreis der Aktivisten rekrutiert wurden. Insgesamt konnte von einer Anleitung der politischen Arbeit in den Kriegsgefangenenlagern durch das Institut 99 keine Rede sein.

\section{Frontbevollmächtigte des Nationalkomitees}

Mit Gründung des Nationalkomitees „Freies Deutschland“ wurden deutsche Kriegsgefangene dauerhaft in die Frontpropaganda der Roten Armee eingebunden. Erste Erfahrungen hatte die 7. Verwaltung im Januar 1943 gesammelt. Von höchster Stelle (Ščerbakov) autorisiert, wurden nicht nur wie bisher kommunistische Emigranten, sondern auch Kriegsgefangene eingesetzt, die die Antifa-Schule absolviert hatten. Die kriegsgefangenen Offiziere Charisius, Hadermann und Reyher wurden an den Kessel von Stalingrad geschickt. Wenig später folgte in Velikie Luki der Einsatz von Leutnant Augustin und den Soldaten Gold, Keßler und Zwiefelhofer. Beide Einsätze waren Pilotversuche der 7. Verwaltung. Während der Einsatz in Stalingrad Frontpropaganda mit herkömmlichen Mitteln - Flugblätter und Appelle über Grabenlautsprecher - erfolgte, wurde der Einsatz in Velikie Luki bewaffnet

63 Puttkamer, Irrtum und Schuld, S. 77.

64 Protokoll der Auflösungssitzung des NKFD vom 2. 11. 1945, in: Verrat hinter Stacheldraht?, S. 261.

65 Weinert an Pieck am 3. 11. 1945, SAPMO-BArch NY 4065/5, Bl. 41.

66 Vgl. Robel, Antifa, S. 92. 
durchgeführt. In den Erinnerungen Keßlers und Golds heißt es euphemistisch, man habe das direkte Gespräch mit dem Kommandierenden des Frontabschnittes suchen wollen, aber es handelte sich vermutlich eher um den Versuch, den kommandierenden Wehrmachtsoffizier durch ein Kommandounternehmen zu töten. Dieses Unterfangen endete ergebnislos in einer Schießerei, wobei es Opfer auf beiden Seiten gab ${ }^{67}$. Auf einem Arbeitstreffen aller 7. Verwaltungen der Fronten im April 1943 wurde über beide Einsätze berichtet. Ziel des Arbeitstreffens war es, „Argumente zu finden, die die Soldaten und Offiziere des Gegners zwingen, die psychologische Barriere zu überwinden und zu kapitulieren"68. Offenbar war sich die Politische Hauptverwaltung noch nicht im Klaren darüber, in welcher Form - als Propagandisten oder als Diversanten - Kriegsgefangene verwendet werden sollten. Durch die sowjetische Entscheidung für das Nationalkomitee „Freies Deutschland“ wurde dem GlavPURKKA die Entscheidung abgenommen. Das Komitee sollte zumindest zu Anfang - mit Argumenten die Wehrmacht zur Aufgabe des Kampfes bewegen. Dessen ungeachtet gab es auch weiterhin die Versuche bewaffneter Einsätze deutscher Kriegsgefangener. Diese wurden zum Teil als Propaganda-, mehr jedoch als Aufklärungs- und teilweise als Kampfeinsatz vom GlavPURKKA immer wieder genutzt ${ }^{69}$.

Das Nationalkomitee stellte eigene Bevollmächtigte für die Frontpropaganda auf. Bei den Nominierungen im Juli/August 1943 wurde dabei auf diejenigen Kriegsgefangenen zurückgegriffen, die ohnehin schon als Propagandisten der 7. Verwaltung tätig gewesen waren. Wahrscheinlich war es sogar so, daß die Nominierungen von Charisius, Reyher und Keßler als Mitglieder des NKFD auf deren Fronteinsätze Anfang 1943 zurückzuführen waren. Keßler wurde eigens zur Gründungsveranstaltung des NKFD von der Front abkommandiert und kehrte nach vier Wochen zur 7. Verwaltung der 2. Belorussischen Front - nun als Frontbevollmächtigter des NKFD - zurück ${ }^{70}$. Bezeichnend für das Verhältnis der Frontbevollmächtigten zum NKFD im Lager Lunevo war auch, daß das Komiteemitglied Charisius nach eigenem Bekunden an keiner Sitzung des Nationalkomitees teilnahm und keines seiner Mitglieder persönlich kannte, da er bis zum Kriegsende ununterbrochen an der Front tätig war ${ }^{71}$. Ebenso war Leutnant Kehler von August bis Dezember 1943 an der Leningrader und von Januar 1944 bis Mai 1945 an der 1. Belorussischen Front, mit deren Stab er nach Berlin einmarschierte ${ }^{72}$. Und als Vizepräsident Emendörfer,

$67 \mathrm{Vgl}$. Keßler, Zur Sache und zur Person, S. 53 ff.; Gold, Im Bunker bei Oberstleutnant von Sass, S. $64 \mathrm{ff}$.

68 Burzew, Einsichten, S. 155.

69 Siehe Wolff, Bewaffnete Gruppen, S. 309 ff.; ders., An der Seite der Roten Armee, S. $67 \mathrm{ff}$., $114 \mathrm{ff} ., 181 \mathrm{ff}$. und $242 \mathrm{ff}$.; Mönicke (Die Teilnahme deutscher Antifaschisten am Partisanenkampf) erfaßt ca. 350 deutsche Kommunisten, Kriegsgefangene und Wehrmachtsdeserteure, die an Kampf- und Propagandaaktionen gegen die Wehrmacht teilnahmen; siehe auch die Erinnerungsberichte in: In den Wäldern Belorußlands, S. $389 \mathrm{ff}$.

70 Keßler, Zur Sache und zur Person, S. 57 und S. 75.

71 Protokoll der Befragung von Charisius, SAPMO-BArch SgY 30/1480, Bl. 12.

72 Kehler, Einblicke und Einsichten, S. $134 \mathrm{ff}$. 
der sich von Juli 1943 bis März 1944 ohne Unterbrechung an der Front aufhielt, von NKFD-Präsident Weinert in das Lager Lunevo zurückbeordert wurde, kannte er dort niemanden, was er in seinen Memoiren in den Satz faßte: „Also war es wirklich notwendig, daß auch ich die Mitarbeiter im Bund kennenlernte."73

Die Organisation der Frontpropaganda lag ausschließlich in der Hand des GlavPURKKA. Marschbefehle und entsprechende Passierscheine der nominell vom NKFD delegierten Frontbevollmächtigten - unabdingbar für die Bewegungsfreiheit im Frontbereich - wurden von der 7. Verwaltung ausgestellt. Die Fahrt zum Einsatzort erfolgte immer in Begleitung eines Politoffiziers, und im Frontbereich unterstanden die Bevollmächtigten direkt der 7. Verwaltung des Frontstabes, mit deren Offizieren sie eng zusammenarbeiteten ${ }^{74}$. Die Verbindung zwischen dem Lager Lunevo und den Frontbevollmächtigten, die zwar auf mehreren Vollsitzungen des NKFD referierten ${ }^{75}$, bestand nur nominell. Lediglich die Kommunisten im Institut 99 (Ulbricht) hatten einen gewissen Überblick über die Aktivitäten des NKFD an der Front. Aber auch ihr Informationsstand hing von den Berichten der Frontbevollmächtigten $\mathrm{ab}$, die zuvor die Zensur des GlavPURKKA durchliefen, unregelmäßig eintrafen und nur relativ oberflächlich waren ${ }^{76}$. Somit blieb das „völlige Informationsmonopol " bei der Politischen Hauptverwaltung77.

Die inhaltliche Ausgestaltung der Frontpropaganda wurde über Direktiven des Rates für militärpolitische Propaganda gesteuert. Manuil'skij hatte bereits im April 1942 als propagandistische Ziele des Komitees formuliert: „Um den unsinnigen Untergang Hunderttausender oder gar von Millionen deutscher Soldaten während der Frühjahrsoffensive [der Roten Armee 1942] zu verhindern, wendet sich das Komitee mit der Losung an alle Soldaten und Offiziere der deutschen Armee, entweder sich sofort von der Front zurückzuziehen und den Rückzug nach Hause anzutreten, oder Bevollmächtigte zu schicken, die mit der Roten Armee über eine kollektive Gefangengabe verhandeln. "78 Im Sommer 1943 entschied sich der Rat für militärpolitische Propaganda für die Losung „Rückführung der Wehrmacht, Rücktritt Hitlers", auf die Manuil'skij die deutschen Kommunisten auf der Zivilsektorsitzung im Institut 99 am 2. August 1943 einschwor ${ }^{79}$. Die damit intendierte Abkehr von einer Überläuferpropaganda ging nicht ohne kontroverse Diskussionen vonstatten. Auf einer zweiten Sitzung im Institut 99 mußte Manuil'skij die deutschen Kommunisten davon überzeugen, daß der Sturz Hitlers nur über ein organisiertes, vom NKFD geleitetes Vorgehen zu erreichen sei. Er schlug - wie sich Pieck notierte - eine "Wendung in der Argumentation" vor. Man sei sich zwar einig, daß

73 Max Emendörfer, Rückkehr an die Front, S. 237; vgl. Jan Emendörfer, Verfemt, S. 154 ff.

$74 \mathrm{Vgl}$. Kommandierung für Bernhard Bechler durch BDO (Seydlitz) und NKFD (Weinert) am 25./26. 4. 1944, SAPMO-BArch SgY 12/1/7, B1. 1 und 7; Frontausweis der 7. Verwaltung für Josef Robiné vom 21.8. 1943, ebenda Bl. 9; Mayenburg, Blaues Blut und rote Fahnen, S. $312 \mathrm{ff}$.; Kopelew, Aufbewahren für alle Zeit, S. 67.

75 Weinert, Nationalkomitee, S. $89 \mathrm{ff}$.

76 Vgl. „Die Arbeit der Frontbevollmächtigten des NKFD“ vom 11. 5. 1944, SAPMO-BArch NY 4065/15, Bl. 48.

77 Vsevolodov, Die propagandistische Tätigkeit des NKFD und BDO, S. 126, siehe auch ebenda S. 131.

78 „Einige Maßnahmen zur Zersetzung der Moral deutscher Soldaten“ vom 3. 4. 1942, RGASPI 495/77/30, Bl. 17.

79 Notizen Piecks „Daten der Bewegung“, SAPMO-BArch NY 4036/575, Bl. 246. 
„Hitler weg muß“, aber es sei besser, die Wehrmacht zu erhalten und geschlossen hinter die deutsche Reichsgrenze zurückzuführen, als sie durch Aufforderung zum Desertieren und Sabotage zu zersetzen ${ }^{80}$.

Durch die von Manuil'skij im Institut 99 in Gang gesetzte Diskussion über die Losung des NKFD wurde der Weg für die Mitarbeit der Offiziere im BDO geebnet, die wiederum notwendig für den Erfolg jeglicher NKFD-Propaganda war. Zeitgleich mit der Diskussion im Institut 99 wurden die Verhandlungen mit den Generälen geführt, bei denen Manuil'skij die Positionen von Generälen und Kommunisten aufeinander abstimmte. Manuil'skijs Anteil an der Formulierung der Propagandalosung ist damit größer, als bisher angenommen ${ }^{81}$. Gemeinsam beschlossen BDO und NKFD auf der vierten Vollsitzung des Nationalkomitees am 24. September 1943 die sogenannte 1. taktische Hauptlosung „Zurückführung der Armee gegen den Befehl Hitlers unter verantwortungsbewußter Führung an die Reichsgrenze". Ab Januar 1944 rief das Nationalkomitee dann doch zum Überlaufen auf und schloß sich damit der Propaganda der 7. Verwaltung an. Auf der sechsten Vollsitzung am 5. Januar 1944 wurde die 2. taktische Hauptlosung „Einstellung des Kampfes, Übergang auf die Seite des Nationalkomitees" beschlossen. Nach dem militärischen Zusammenbruch der Wehrmacht an der Ostfront im Sommer 1944 wurde diese Losung noch durch den Zusatz „Alle Waffen gegen Hitler" ergänzt ${ }^{82}$.

Für die Politische Hauptverwaltung war die Einbindung der nominell eigenständigen Propaganda des NKFD in die Strukturen der 7. Verwaltung nicht unproblematisch. Von Anfang an machte man den Versuch, die Parolen des Nationalkomitees und die der Roten Armee getrennt voneinander zu halten. Wenige Tage nach der Gründung des Nationalkomitees hatte das GlavPURKKA per Direktive gegenüber den Politoffizieren klargestellt, daß die Aufrufe des NKFD von „nationalen deutschen Interessen" ausgehen, die Propaganda der 7. Verwaltung dagegen „die Erfolge der Roten Armee herausstellen, auf die Unvermeidlichkeit der Niederlage von Hitler und auf Aufrufe der freiwilligen Gefangengabe von Offizieren und Soldaten Gewicht legen" solle ${ }^{83}$. War somit für die Offiziere der 7. Verwaltung durchgängiges Motto, daß Deutsche zu Deutschen sprechen sollten ${ }^{84}$, so scheiterte dieses Konzept dennoch, weil die Methoden und Organisationsformen der Frontpropaganda immer die des GlavPURKKA blieben: „Letztlich hat die Propaganda des NKFD in keiner Form gefruchtet. Was bleibt? Die Ziele des NKFD, soweit sie sich aus seinen ,Bildern im Kopf' beurteilen lassen, scheinen rein, manche Mittel seiner Propaganda dagegen weniger: die euphemistisch gezeichneten Feinde [die Sowjetunion], der irreale Schatten einer übergehenden Wehrmacht, das Märchen von der starken Freiheitsbewegung NKFD. Und wenn es richtig ist, daß man Menschen

80 Notizen Piecks vom 9. 8. 1943, SAPMO-BArch NY 4036/498, Bl. 87: „Daß Hitler weg muß = einig, aber wie, anderer Führer - Wehrmacht erhalten und geschlossen in die Heimat zurück, Kampfausschüsse $=$ nicht einverstanden - Kampfgruppe Ausweg - betr[ogene (?)] Demokratie“.

81 Scheurig (Verräter oder Patrioten, S. 69) und Sywottek (Deutsche Volksdemokratie, S. 129) führen die Formulierung der Losung allein auf den Einfluß des BDO zurück. Diesener (Propagandaarbeit, S. 34) versucht, den Einfluß der KPD auf die Formulierung herzuleiten und spricht von einem Kompromiß zwischen KPD und BDO.

$82 \mathrm{Zu}$ den Vollsitzungen des NKFD siehe Weinert, Nationalkomitee, S. $88 \mathrm{ff}$.

83 Vsevolodov, Die propagandistische Tätigkeit des NKFD, S. 121.

84 Kopelew, Aufbewahren für alle Zeit, S. 62 f. 
nicht nur nach ihren Zielen beurteilen darf, sondern auch nach den Mitteln, die sie zur Erreichung ihrer Ziele einsetzen, dann bleiben zumindest Fragezeichen." 85 Vermutlich waren die Losungen des NKFD und die des GlavPURKKA für den deutschen Frontsoldaten von Anfang an kaum auseinanderzuhalten, zumal ab 1944 von beiden zum Überlaufen aufgefordert wurde.

Die Hauptaufgabe des Instituts 99 bestand darin, die Unterstützung der Frontpropaganda der Roten Armee durch das NKFD zu steuern. Als Verbindungsmann hatte das GlavPURKKA den stellvertretenden Chef der 7. Verwaltung, Oberst Braginskij, zum Institut 99 abgestellt ${ }^{86}$. Für die kriegsgefangenen Mitglieder des Nationalkomitees trat Walter Ulbricht als Leiter der operativen Abteilung und somit als der entscheidende Mann auf87. Typisch ist die Wahrnehmung von Hermann Rentzsch, der nach dem Besuch der Antifa-Schule an die 1. Baltische Front entsandt wurde: Er wurde „von Walter Ulbricht in seiner Eigenschaft als Mitglied des Nationalkomitees ,Freies Deutschland' und Leiter der Operativ-Abteilung vereidigt" 88 .

Die Besetzung der Frontbevollmächtigten blieb bis Kriegsende relativ konstant. Die meisten waren im August 1943 an die Front gekommen oder hatten schon vorher mit der 7. Verwaltung zusammengearbeitet. Für die Bevollmächtigten Kehler, Fleschhut, Klein, Charisius und Stresow läßt sich zudem belegen, daß sie mit der Roten Armee nach Deutschland kamen ${ }^{89}$. Es wurden nicht nur Frontbevollmächtigte entsandt, sondern zu deren Unterstützung auch nach und nach sogenannte Beauftragte und Helfer. Die Stärke der stetig wachsenden Frontorganisation des NKFD läßt sich schwer abschätzen. Im Juli und August 1943 wurden 13 Frontbevollmächtigte des NKFD ernannt und durch die Gründung des BDO zusätzlich vier BDO-Mitglieder (Einsiedel, von Kügelgen, Gudzent, Müller) an die Fronten delegiert ${ }^{90}$. Auf der fünften Vollsitzung des NKFD am 19. November 1943 berichtete Vizepräsident Zippel von 12 ständigen Bevollmächtigten (der Bevollmächtigte Kühn war gefallen), 120 Beauftragten und zusätzlich „einzelnen Helferbrigaden“91. Im Herbst 1943 hielten sich ungefähr 150 NKFD-Propagandisten im Frontbereich auf ${ }^{92}$. Im April 1944 beschloß das Plenum des Nationalkomitees im Rahmen seiner

85 Starkulla, Verderber Hitler - preisgegebenes Deutschland, S. 69.

86 Vsevolodov, Die propagandistische Tätigkeit des NKFD, S. 122.

87 Aussagen von Seydlitz' in einer Befragung durch das Militärgeschichtliche Forschungsamt im März 1969; BArch-MA N 55/22, Bl. 21 u. 31; vgl. Scheurig, Verräter oder Patrioten, S. $95 \mathrm{f}$.

88 Rentzsch, Es war die Schule meines Lebens, S. 273.

89 Notizen Piecks auf der Aufstellung über "Kader der KPD in der Sowjetunion" vom 30. 5. 1945, in: „Gruppe Ulbricht“, S. 441.

90 Wolff, An der Seite der Roten Armee, S. 22. Einsiedel war von Mitte Oktober bis Mitte Dezember 1943 und von Anfang Januar bis Anfang März 1945 erst an der 4. Ukrainischen, dann an der 2. Belorussischen Front, Einsiedel, Tagebuch der Versuchung, S. $101 \mathrm{ff}$. und S. $168 \mathrm{ff}$. Von Kügelgen wurde im September 1943 an die 1. Ukrainische Front delegiert, Kügelgen, Nacht der Entscheidung, S. $402 \mathrm{ff}$.

91 Weinert, Nationalkomitee, S. 89. Wolff (An der Seite der Roten Armee, S. 24 u. 274 f.) nennt 17 Bevollmächtigte und 43 Armeebeauftragte. Da der Frontbevollmächtigte Kühn fiel, wird die $Z$ ahl von 12 Bevollmächtigten stimmen.

92 Die von Wolff (An der Seite der Roten Armee, S. 274 f.) geschätzte Zahl von 350 bis 400 ist zu hoch gegriffen. Wolff geht von 17 Frontbevollmächtigten, 43 Armeebeauftragten von den zentralen Antifa-Schulen, 150 Helfern von den Frontschulen, ca. 100 neuen Absolven- 
achten Vollsitzung die Nominierung weiterer Bevollmächtigter, die aber erst in Angriff genommen wurde, nachdem sich die 7. Verwaltung für den Ausbau der Frontorganisation des NKFD ausgesprochen hatte ${ }^{93}$.

Aufstellung der 7. Verwaltung über die Frontbevollmächtigten des NKFD 94 (September 1944)

\begin{tabular}{|c|c|c|}
\hline Front & Bevollmächtigter & seit \\
\hline $\begin{array}{l}\text { Karelische } \\
\text { Leningrader } \\
\text { 3. Baltische } \\
\text { 2. Baltische } \\
\text { 1. Baltische } \\
\text { 3. Belorussische } \\
\text { 2. Belorussische } \\
\text { 1. Belorussische } \\
\text { 1. Ukrainische } \\
\text { 2. Ukrainische } \\
\text { 3. Ukrainische } \\
\text { 4. Ukrainische }\end{array}$ & $\begin{array}{l}\text { Oberleutnant Robiné } \\
\text { Leutnant Kehler } \\
\text { Feldwebel Baindl } \\
\text { Leutnant Willms } \\
\text { Soldat Fleschhut } \\
\text { Obergefreiter Gold } \\
\text { Obergefreiter Gold } \\
\text { Leutnant Willms } \\
\text { Unteroffizier Klein } \\
\text { Oberst Steidle } \\
\text { Leutnant Bürk } \\
\text { Oberleutnant Charisius } \\
\text { Feldwebel Stresow }\end{array}$ & $\begin{array}{l}\text { August } 1943 \\
\text { September } 1943 \\
\text { Aug. 1943 - März. 194495 } \\
\text { August194396 } \\
\text { August 1944 } \\
\text { Juli 1943 - Aug. 194497 } \\
\text { März 1944 } \\
\text { Mai 194498 } \\
\text { August } 1943 \\
\text { August } 1943 \\
\text { August } 1943\end{array}$ \\
\hline
\end{tabular}

Mitte Mai 1944 kamen die Politoffiziere der 7. Verwaltungen der Fronten zu dem zweiten sogenannten Armeetreffen zusammen, auf dem die Ausweitung der Frontorganisation des NKFD zweifellos Hauptthema war99. Wie die Schlappe am Kessel von Korsun' deutlich vor Augen geführt hatte, war der erhoffte propagandistische Erfolg des Nationalkomitees ausgeblieben. Zudem hatten die Pläne des BDO über die Bildung einer Exilregierung für viel Aufregung gesorgt. Ščerbakov versuchte daher in seiner Eigenschaft als Chef des GlavPURKKA, den Politoffizieren die Haltung der Politischen Hauptverwaltung zu verdeutlichen ${ }^{100}$. Nun wurde die

ten der Frontschulen und 50-100 Propagandisten in den Frontsammellagern sowie von technischem Personal aus. Da jedoch die Frontschulen erst mit ihrer Schulungstätigkeit begannen, können die von Wolff angenommenen Absolventenzahlen (insgesamt 250) nicht so hoch gewesen sein. Vermutlich wurde im Herbst 1943 vornehmlich auf die Antifa-Schüler aus dem Hinterland zurückgegriffen.

93 Weinert, Nationalkomitee, S. 92; Seleznev an Manuil'skij am 26.5.1944, RGASPI 495/77/38, Bl. 13.

94 RGVA/K 88/3/1, Bl. 76.

95 „Die Arbeit der Frontbevollmächtigten des NKFD“ vom 11. 5. 1944, SAPMO-BArch NY 4065/15, B1. 51.

96 Ebenda.

97 Ebenda; Wolff, An der Seite der Roten Armee, S. 22.

98 Steidle wurde im Februar 1945 von der Front abberufen, Steidle, Entscheidung an der Wolga, S. 360.

99 Burzew (Einsichten, S. 83 ff., S. 155 ff. und S. 214 ff.) berichtet von Treffen dieser Art im Mai 1942, April 1943 und Mai 1944. Offensichtlich gab es so etwas wie ein Frühjahrstreffen der 7. Verwaltungen der Fronten.

100 „Scherbakow erinnerte daran, daß man die zwei Richtungen in der Propaganda, nämlich die der Roten Armee und die des NKFD, nicht vermengen dürfe (...)“, ebenda, S. $216 \mathrm{f}$. 
Frontorganisation des NKFD erheblich ausgebaut. UPVI und GlavPURKKA einigten sich auf einen Auswahlmodus für die Kriegsgefangenen, an dem das Institut 99 nur mittelbar beteiligt war ${ }^{101}$. Die Kaderentscheidung erfolgte analog zu der Ernennung eines Lagerbevollmächtigten des NKFD: Auf Vorschlag der Politabteilung des UPVI stellte das Institut 99 die notwendigen Personalunterlagen zusammen, mit denen die Nominierung an die 7. Verwaltung weitergereicht wurde ${ }^{102}$. Somit hatte das Institut zumindest den Überblick, wer aus den Kriegsgefangenenlagern bzw. den Antifa-Schulen - in der Regel mußten alle Frontpropagandisten die Antifa-Schule absolviert haben ${ }^{103}$ - an die 7. Verwaltung überstellt wurde. Die Frontorganisation des NKFD wurde hierarchisch gegliedert. Dem Bevollmächtigten waren sogenannte Beauftragte unterstellt, die ihrerseits Helfer auf Armee- (eine Front der Roten Armee bestand aus bis zu 15 Armeen) und Divisionsebene einsetzten. Das Einsetzen von Beauftragten und Helfern hatte bereits Ende 1943 begonnen, wurde aber erst nach der Abstimmung des GlavPURKKA mit UPVI und Institut 99 im Frühjahr 1944 forciert. Die nun vermehrt benötigten Kader wurden nur zum Teil über die Antifa-Schulen ausgewählt. Ab der zweiten Jahreshälfte 1944 wurden sie zunehmend über die Frontschulen der 7. Verwaltung rekrutiert.

Antifaschistische Frontschulen waren seit dem Frühjahr 1943 an einigen Fronten der Roten Armee erprobt worden. Im März 1943 hatte die 7. Verwaltung an der 2. Baltischen Front (Nordwestfront) damit begonnen, Kriegsgefangene im Frontbereich politisch zu schulen, um damit ein Reservoir an deutschen Propagandisten zu bilden. Aus diesen Vorbereitungskursen entwickelten sich im Laufe des Jahres 1943 zuerst an der 2. Baltischen Front, dann an der Leningrader sowie der 3. und 4. Ukrainischen Front die ersten antifaschistischen Frontschulen. Vermutlich wurde auf der Armeeberatung im Mai 1944 der Ausbau der Frontschulen beschlossen, denn im Sommer 1944 kamen weitere Schulen an der 1. Baltischen, der 2. und 3. Belorussischen und der 1. Ukrainischen Front hinzu1 ${ }^{104}$. Mit Ausnahme der 1. Belorussischen Front wurden an jeder Front Schulungen durchgeführt.

Mit der Ausweitung der antifaschistischen Frontschulen einher ging offenbar auch eine Erweiterung der Aufgaben für die dort ausgebildeten Kader. Die Frontverwaltungen des GlavPURKKA hatten bereits Ende 1943 damit begonnen, im Namen des NKFD Kriegsgefangene als Diversanten im Rücken der Wehrmacht einzusetzen ${ }^{105}$. Im Februar 1944 wurden erstmals Absolventen der Antifa-Schule per Fallschirm weit hinter den deutschen Linien abgesetzt. Die „Gruppe 117“ unter der Leitung zweier sowjetischer Offiziere hatte den Auftrag, in der Etappe der Wehrmacht eine Untergrundorganisation, eine „Wehrmachtgruppe des NKFD“, aufzubauen. Die Kriegsgefangenen waren mit deutschen Uniformen, Waffen und gefälschten Papieren sowie mit Funkgerät und Druckerpresse zur Herstellung von

101 „Bestimmung über die Ausnutzung der Kriegsgefangenen NKFD- und BDO-Bevollmächtigten an den Fronten zur Zersetzung der gegnerischen Truppen", ohne Datum [vermutlich Mai 1944], RGASPI 495/77/42, B1. 73.

102 Jakovec an Dimitrov am 26. 8. 1944 und Mirov (Kaderabteilung des OMI) an Kozlov am 8. 9. 1944, RGASPI 495/77/47, Bl. 34-35; vgl. ebenda Bl. 63-64 den analogen Vorgang.

103 Robel, Antifa, S. 87.

104 Wolff, An der Seite der Roten Armee, S. 24 u. 178; Burzew, Einsichten, S. 196; siehe auch Engelbert, Schule des Propagandisten, S. $108 \mathrm{ff}$.

105 Wolff, An der Seite der Roten Armee, S. $117 \mathrm{ff}$. 
Flugblättern ausgestattet worden. Es gelang ihnen, unerkannt bis zum Zusammenbruch der Heeresgruppe Mitte im Juli 1944 ihrer Arbeit nachzugehen, um danach unversehrt nach Moskau zurückzukehren ${ }^{106}$.

Der Ausbau der Schulen an nahezu jeder Front hing vermutlich mit der Verstärkung der Diversionseinsätze im Rücken der Wehrmacht zusammen. So ordnete das GlavPURKKA anläßlich der Gründung der Schule an der 4. Ukrainischen Front im Januar 1944 an: „Das Programm der Schule ist auf eineinhalb bis zwei Monate Dauer auszulegen. Ihr Ziel ist die Vorbereitung von Antifaschisten, die in der Lage sind, entsprechende Aufgaben im Hinterland der deutschen Armee auszuführen und an der schriftlichen und mündlichen Propaganda zur Zersetzung der Truppen des Feindes beizutragen." 107 Durch die Frontschulen wuchs ein Kreis von Aktivisten heran, der nach erfolgreichen Einsätzen an die Frontschule zurückkehrte und weitergeschult wurde. So erhielten die Frontschulen den einmal aufgebauten Kaderstamm ${ }^{108}$. An der 1. Ukrainischen Front waren es beispielsweise im Schnitt 120 Aktivisten, d.h. zentral vom Institut 99 eingesetzte Frontbevollmächtigte und Beauftragte des NKFD sowie über die Frontschulen der 7. Verwaltung rekrutierte Helfer, die für den Einsatz im Hinterland ausgewählt wurden ${ }^{109}$.

Es ist nicht eindeutig zu sagen, wie viele Kurse in welcher Stärke an den Frontschulen durchgeführt wurden. An der Schule der 2. Baltischen Front, der ersten ihrer Art, fanden mindestens fünf Durchläufe statt. Den ersten Kurs schlossen im August 1943 zweiundzwanzig und den vierten Kurs im September 1944 vierzehn Kriegsgefangene $a b^{110}$. Eine Aufstellung vom April 1945 erfaßt insgesamt 337 Schüler an sechs Fronten, wobei allein an der 1. Ukrainischen Front 100, dagegen an der 3. Ukrainischen nur 19 Kriegsgefangene geschult wurden ${ }^{111}$. Wolff nimmt an, daß im Sommer 1944 die Frontorganisation des NKFD auf ca. 1000 Kriegsgefangene ausgeweitet wurde. Im Herbst 1944 sollen es bereits 1500 Kriegsgefangene gewesen scin, und bis Kriegsende habe sich die Stärke der Frontorganisation des NKFD auf 1800 bis 2000 Mann belaufen ${ }^{112}$. Auch wenn Wolffs Schätzungen mit Vorsicht zu begegnen ist, da er die nur wenigen Angaben zu einzelnen Fronten hochrechnet, so ist doch unbestritten, daß die Zahl der deutschen Frontpropagandisten 1944/45 um ein Vielfaches wuchs.

Ab dem Frühsommer 1944 leiteten die Bevollmächtigten des NKFD eine personell stetig anwachsende Gruppe von Propagandisten und übernahmen zahlreiche

106 Ebenda, S. 123 ff.; Burzew, Einsichten, S. 225 f.; Krohn, „Wir schossen mit Flugblättern...“, S. $32 \mathrm{ff}$;; Bahrs, In den Wäldern Belorußlands, S. $317 \mathrm{ff}$.

107 Zitiert nach Pogrebnoj, Dejatel'nost' nemeckich antifašistov, S. 163.

108 Es bleibt in diesem Zusammenhang offen, wie hoch bei den Fronteinsätzen die Verluste waren, die es zweifellos gegeben hat. Weinert sprach bei der Auflösung des NKFD von „Hunderten von Toten“, Protokoll der Auflösungssitzung des NKFD am 2.11. 1945, in: Verrat hinter Stacheldraht?, S. 262.

109 Dohm, Zur Arbeit der Frontschule, S. 57; vgl. Eildermann, Die Antifa-Schule, S. 29.

110 MMNA 822/173; BArch-MA TS-1, Dok. 81.

111 1. Ukrainische: 100, 3. Ukrainische: 19 , 4. Ukrainische: 48, 2. Belorussische: 51 , 1. Baltische: 77 und Leningrader Front: 42 Schüler; Vsevolodov, Vzaimodejstvie politorganov, Anlage Nr. 17.

112 Wolff, An der Seite der Roten Armee, S. 94, 150 und 213f. Wolffs Angaben zu den Kursstärken sind ungenau. Er veranschlagt 25-150 Absolventen pro Kurs, ohne dies näher zu spezifizieren, ebenda S. 180. 
Aufgaben von der 7. Verwaltung der Front. Sie schrieben Flugblätter und führten Lautsprechersendungen in den vordersten Schützengräben durch. Sie waren bei der Befragung neuer Kriegsgefangener dabei und erstellten für die 7. Verwaltung Analysen der „politisch-moralischen Stimmung unter den Soldaten des Gegners“. Sie rekrutierten neue Propagandisten, indem sie kleinere Propagandaaufträge verteilten: Neue Gefangene wurden aufgefordert, über Lautsprecher zu ihren Kameraden zu sprechen oder ein Flugblatt zu schreiben. Auch wurden Neu-Gefangene auf die deutsche Seite zurückgeschickt, um dort ihre Kameraden dazu zu bewegen, sich freiwillig in Gefangenschaft zu begeben. Zugleich sollte mit dem Zurückschicken bewiesen werden, daß Gefangene nicht erschossen werden. Ferner halfen die Frontpropagandisten des NKFD dabei, Briefe deutscher Kriegsgefangener in die Feldpost der Wehrmacht einzuschleusen, um damit in der Heimat ein Lebenszeichen zu geben ${ }^{113}$. Den Frontbevollmächtigten selbst kam mit Ausweitung der Frontorganisation mehr und mehr die Aufgabe der Kontrolle und der Kaderrekrutierung zu. In diesem Zusammenhang wurden die Frontschulen, an denen die Bevollmächtigten zusammen mit Offizieren der 7. Verwaltung und deutschen Kommunisten ${ }^{114}$ unterrichteten, zunehmend wichtiger.

Das Programm der antifaschistischen Frontschulen, das sich im allgemeinen an dem in den Antifa-Schulen vermittelten Curriculum orientierte ${ }^{115}$, wandelte sich im Herbst 1944. Nun wurden konkrete Fragen der Besatzungsverwaltung einbezogen. Aber wie bei allen politischen Schulungen war der Anteil allgemein historisch-politischer Themen sehr groß, so daß sich der inhaltliche Wechsel lediglich an den Abschlußlektionen bemerkbar machte. Im vierten Kurs an der 2. Baltischen Front (August 1944) wurden folgende Themen durchgearbeitet: (1) „Die Lehren aus drei Jahren Krieg Hitler-Deutschlands gegen die UdSSR. Rolle und Bedeutung des NKFD“, (2) „Die Lehren aus der Geschichte Deutschlands“, (3) „Hitler als Kriegsschuldiger", (4) „Die Wahrheit über die Sowjetunion“ und (5) „Hitler hat Deutschland in die Katastrophe geführt - wo ist der Ausweg für das deutsche Volk? "116. Für den darauffolgenden fünften Kurs, der im September 1944 begann, standen die gleichen Themen auf dem Programm. Allerdings erfolgte eine Erweiterung um die beiden Abschlußlektionen „Die Gesetze der sowjetischen Regierung über die Behandlung von Kriegsgefangenen" und „Wesen und Charakter der sowjetischen Propaganda gegenüber der deutschen Armee und der deutschen Bevölkerung "117. Die Frontschulen konnten allerdings nur so arbeiten, wie es das Kampfgeschehen erlaubte. Häufige Propagandaeinsätze der Schüler und ständiges Umziehen zusammen mit dem Frontstab brachten immer wieder Unterbrechungen in den Schulablauf. So wechselte die Schule an der 1. Ukrainischen Front von März 1944 bis Mai

113 Vgl. den Tätigkeitsbericht von Eberhard Charisius an der 4. Ukrainischen Front, RGASPI 495/77/42, Bl. 55-72. Im Frühjahr 1944 waren an der 4. Ukrainischen Front 3 Beauftragte und 11 Helfer eingesetzt.

114 Helene Berner an der 2. Baltischen, Bernhard Dohm an der 1. Ukrainischen und Wilhelm Eildermann an der 3. Ukrainischen Front.

115 Eildermann, Die Antifa-Schule, S. 51 ff.; Berner, Mit der Sowjetarmee nach Berlin, S. 325; vgl. Weinert, Nationalkomitee, S. 54.

116 BArch-MA TS-1, Dok. 82.

117 Ebenda, Dok. 81. 
1945 fünfmal ihren Standort. In dieser Zeit erfolgten „273 Einsätze“118. Die häufigen Einsätze der Schüler führten zu einer Zweiteilung der Schulung. In einem Kurzlehrgang wurde auf konkrete Aufgaben der Frontpropaganda, wie z.B. das Schreiben von Flugblättern, vorbereitet, während der eigentliche Antifa-Kurs als „Vertiefung der bereits erworbenen Kenntnisse" galt ${ }^{119}$. An der 2. Baltischen Front dagegen herrschten ab dem Jahreswechsel 1944/45 relativ stabile Bedingungen, die eine kontinuierliche Schulung zuließen ${ }^{120}$.

Anfang 1945 wurden die Schule der 2. Baltischen und die der 1. Baltischen Front in der Nähe von Stettin zusammengelegt. Dort bezog die Frontschule auch die Zivilbevölkerung (Frauen) in ihre Arbeit ein, um Einfluß auf die Flüchtlingstrecks aus Ostpreußen zu bekommen ${ }^{121}$. Im April 1945 schließlich wurde die Frontschule in den Befehlsbereich der 1. Belorussischen Front, die vermutlich nie eine eigene Frontschule gehabt hatte, an den östlichen Stadtrand von Berlin nach Rüdersdorf verlegt ${ }^{122}$. Von dort aus wurden Ende April zwölf NKFD-Bevollmächtigte auf Berlin verteilt ${ }^{123}$. Andere Absolventen wurden in den folgenden Wochen abberufen, um als Bürgermeister oder Landräte in den von der Roten Armee besetzten Gebieten die kommunalen Verwaltungen in Gang zu bringen. In Vorpommern und Mecklenburg waren über die 7. Verwaltung an der 2. Belorussischen Front 18 Kriegsgefangene eingesetzt worden, die damit alle Verwaltungszentren besetzen konnte $^{124}$. Auf diesen Nutzen der Frontschulen für die Besatzungsverwaltung der Roten Armee in Deutschland hatte Oberst Tjul'panov - seinerzeit Leiter der 7. Verwaltung an der 4. Ukrainischen Front, nach dem Krieg Chef der Propagandaabteilung der SMAD - bereits auf der Armeeberatung im Mai 1944 hingewiesen: „Zum Beispiel ist es außerordentlich wichtig, eine Gruppe zuverlässiger Antifaschisten zu seiner Verfügung zu haben, die aus den Gebieten stammen, in die in nicht all zu ferner Zukunft die Truppen dieser oder jener Front einmarschieren werden. Weil es aber unter Umständen schwierig sein wird, die benötigten Leute an den Frontschulen auszuwählen, scheint es mir unerläßlich zu sein, schon jetzt in den Lagern im Hinterland oder in zentralisierter Form damit zu beginnen, solche Gruppen vorzubereiten." ${ }^{25}$ Sprachen Tjul'panovs Anregungen vom Mai 1944 noch die Möglichkeit an, die Kaderrekrutierung über das Institut 99 zu zentralisieren, so stellte Pieck im Dezember 1944 gegenüber Dimitrov fest: „Der Vorschlag, an der Front überprüfte Antifaschisten für unseren Einsatz zu verwenden, scheint uns kaum durchführbar, da die Rote Armee diese Kader selbst einsetzt und wir ein Interesse daran haben, daß von der Front aus möglichst viele zum Einsatz kommen. Unsere Aufgabe muß darin bestehen, daß in den Kriegsgefangenenlagern (Arbeitslagern)

118 Dohm, Zur Arbeit der Frontschule, S. 57.

119 Dohm, Schule der guten Deutschen, S. $390 \mathrm{f}$.

120 Die Rote Armee hatte sich bis an das Westufer der Düna vorgekämpft und blieb bis zum Kriegsende am sogenannten Kurlandkessel westlich von Riga stehen, Sovetskaja voennaja énciklopedija, tom 6, S. 519.

121 Wolff, An der Seite der Roten Armee, S. 233 f. und S. 255.

122 Vsevolodov, Vzaimodejstvie politorganov, S. 126; Wolff, An der Seite der Roten Armee, S. 211 und S. 262; Berner, Mit der Sowjetarmee nach Berlin, S. $329 \mathrm{ff}$.

123 Rentzsch, Mein Weg zum Frontbeauftragten, S. 415 ff.; Kehler, Einblicke und Einsichten, S. 224.

124 Ebenda, S. 257 f.; Wolff, An der Seite der Roten Armee, S. 257 f.

125 Vortrag Tjul'panovs vom 4. 5. 1944, RGASPI 495/77/42, Bl. 72. 
bewährte Kommunisten und Antifaschisten ausgewählt und zur Prüfung an der Front oder für die Schule [Antifa-Schule] vorgeschlagen werden."126

Im Zuge der Kaderrekrutierung für Besatzungsaufgaben in Deutschland verlor das Institut 99 seinen Einfluß auf die Frontorganisation des NKFD, denn das GlavPURKKA wollte die Einmischung anderer Institutionen vermeiden. Vor allem wollte es das Nationalkomitee nicht mit der sowjetischen Besatzungsverwaltung in Berührung kommen lassen. Um den eigenen Apparat besser auf die kommenden Besatzungsaufgaben vorzubereiten, wurde im Sommer 1944 die 7. Verwaltung umstrukturiert. Es entstand die Unterabteilung „Vorbereitung und Verwendung von Antifaschisten"127. Außerdem schulte das GlavPURKKA von Mitte November 1944 bis Anfang Mai 1945 in eigener Regie ihre Politoffiziere ${ }^{128}$. Ab dem Herbst 1944 gingen Institut 99 und GlavPURKKA in der Vorbereitung auf den Einsatz in Deutschland getrennte Wege. Symptomatisch für das daraus resultierende Spannungsverhältnis war die Beendigung des Einsatzes des NKFD-Frontbevollmächtigten Kehler. Er hatte seit Gründung des Nationalkomitees ununterbrochen an der Front gearbeitet und war über die Frontschule Rüdersdorf nach Berlin gekommen. Dort hatte er von der 7. Verwaltung den Auftrag bekommen, das Bürgermeisteramt von Berlin-Mitte einzurichten, wozu er ein Haus in der Berliner Parochialstraße requirierte. Vollkommen überraschend - so Kehler in seinen Memoiren - tauchte eines abends der über das Institut 99 nach Deutschland geschickte Walter Ulbricht auf und erklärte ihm: „Also erstens ist das nicht das Haus des Bezirksbürgermeisters von Mitte, sondern der Sitz des künftigen Oberbürgermeisters von Berlin. (...) Zweitens bist du nicht mehr Frontbevollmächtigter des Nationalkomitees ,Freies Deutschland" an der 1. Belorussischen Front."129

Die Anleitung der Frontbevollmächtigten des NKFD durch das Institut 99 bestand nur nominell. Seit dem Frühjahr 1944 verlor es mehr und mehr den direkten Einfluß auf die als Propagandisten an die Front entsandten Antifaschisten. Erst bei Kriegsende konnte das Institut 99 durch die aus Moskau geschickten Kadergruppen („Gruppe Ulbricht") unmittelbaren Einfluß auf die Geschehnisse im Frontbereich, d. h. die Anfänge der sowjetischen Besatzungsverwaltung, nehmen. Der Ausbau der Antifa-Schulen in den Kriegsgefangenenlagern des sowjetischen Hinterlandes und der Einsatz ihrer Absolventen erwies sich somit als das zweite Standbein des Instituts 99.

126 Pieck an Dimitrov am 9. 12. 1944, in: „Gruppe Ulbricht“, S. 137.

127 Vsevolodov, Vzaimodejstvie politorganov, Anlage Nr. 3; Burzew, Einsichten, S 236.

128 Vsevolodov, Vzaimodejstvie politorganov, S. 142; vgl. Creuzberger, Die sowjetische Besatzungsmacht, S. $22 \mathrm{ff}$.

129 Kehler, Einblicke und Einsichten, S. 239. 